\title{
A pilot study of patient-centered outcome assessment using PROMIS for patients undergoing colorectal surgery
}

\author{
Traci L. Hedrick ${ }^{1}$ - Amy M. Harrigan ${ }^{1}$ • Robert H. Thiele ${ }^{2}$ - Charles M. Friel ${ }^{1}$ • \\ Benjamin D. Kozower ${ }^{3} \cdot$ George J. Stukenborg ${ }^{4}$
}

Received: 10 August 2016 / Accepted: 17 April 2017 / Published online: 24 April 2017

(C) The Author(s) 2017. This article is an open access publication

\begin{abstract}
Purpose Few studies have assessed patient-reported outcomes following colorectal surgery. The absence of this information makes it difficult to inform patients about the nearterm effects of surgery, beyond outcomes assessed by traditional clinical measures. This study was designed to provide information about the effects of colorectal surgery on physical, mental, and social well-being outcomes.

Methods The NIH Patient-Reported Outcomes Measurement Information System (PROMIS®) Assessment Center was used to collect patient responses prior to surgery and at their routine postoperative visit. Four domains were selected based on patient consultation and clinical experience: depression, pain interference, ability to participate in social roles and activities, and interest in sexual activity. Multilevel random coefficient models were used to assess the change in scores

Results from this research have been presented in part at the PROMIS Health Organization Inaugural Conference, on May 15, 2015, in Philadelphia PA, USA, and at the American Society of Colon and Rectal Surgeons Annual Scientific Meeting on May 1, 2016 in Los Angeles, CA, USA.
\end{abstract}

Electronic supplementary material The online version of this article (doi:10.1007/s00520-017-3718-4) contains supplementary material, which is available to authorized users.

Traci L. Hedrick

TH8Q@hscmail.mcc.virginia.edu

Amy M. Harrigan

ACM6A@hscmail.mcc.virginia.edu

Robert H. Thiele

RHT7W@virginia.edu

Charles M. Friel

CMF2X@hscmail.mcc.virginia.edu

Benjamin D. Kozower

kozowerb@wudosis.wustl.edu during the follow-up period and to assess the statistical significance of differences in trends over time associated with key clinical measures.

Results In total, 142 patients were consented, with 107 patients completing pre- and postoperative assessments (75\%). Preoperative assessments were typically completed 1 month prior to surgery (mean 29.5 days before, $\mathrm{SD}=19.7$ ) and postoperative assessments 1 month after surgery (mean 30.7 days after, $\mathrm{SD}=9.2$ ), with a mean of 60.3 days between assessment dates. Patients demonstrated no statistically significant changes in scores for pain interference $(-0.18$ points, $p=0.80)$ or the ability to participate in social roles and activities ( 0.44 points, $p=0.55)$, but had significant decreases in depression scores between pre- and postoperative assessments ( -1.6 points, $p=0.03$ ) and near significant increases in scores for interest in sex $(1.5$ points, $p=0.06)$. Pain interference scores for

George J. Stukenborg

gstukenborg@virginia.edu

1 Department of Surgery, University of Virginia Health System, Charlottesville, VA, USA

2 Department of Anesthesiology, University of Virginia Health System, Charlottesville, VA, USA

3 Division of Cardiothoracic Surgery, Department of Surgery, Washington University, St. Louis, MO, USA

4 School of Medicine, University of Virginia Health System, P.O. Box 800717, Charlottesville, VA 22908-0821, USA 
patients with neoadjuvant chemotherapy significantly increased (3.5 points, $p=0.03$ ). Scores for the interest in sex domain decreased (worsened) for patients with oncologic etiology $(-3.7$ points, $p=0.03)$. No other differences in score trends by patient characteristics were large enough to be statistically significant at the $p<0.05$ threshold.

Conclusion These data suggest that the majority of patients quickly return to baseline physical, mental, and social function following colorectal surgery. This information can be used preoperatively to counsel patients about the typical impact of colorectal surgery on quality of life.

Keywords Patient reported outcomes · PROMIS · Colorectal surgery $\cdot$ Enhanced recovery after surgery

\section{Introduction}

Mortality, morbidity, and length of stay are key clinical outcomes for patients following colorectal surgery. Outcomes that reflect the patients' subjective perspective, including depression, pain interference, and an individual's ability to function socially, are also of great significance, though these outcomes are less frequently investigated [1].

These other physical, mental, and social function domains of health are examples of patient-reported outcomes (PROs), which taken together comprise the overall quality of life obtained following treatment [2]. The collection and evaluation of PROs are of growing interest for the assessment of the overall quality of life obtained by patients following treatment and also for guiding patient decision-making and symptom management [3]. Implementation of methods for assessing PROs in surgical care has advanced most quickly for total joint replacement patients [4, 5]. The Centers for Medicare and Medicaid (CMS) recently announced plans to collect preoperative and postoperative PROs for patients with lower extremity joint replacement procedures, as a component of payment reforms for Medicare beneficiaries [6]. New initiatives that will require the collection of PROs for patients with other commonly performed surgical procedures are anticipated, primarily through the modification of existing surgery patient registries [7].

A more complete understanding of patient outcomes experienced following colorectal surgery requires the assessment of PROs. There are several different types of measurement tools for evaluating patient-centered outcomes. A systematic review of the PROs that have been used in colorectal cancer surgical studies indicates that there is significant heterogeneity in methods for measuring psychological, symptomatic, and functional domains [8].

Standardized tools for the measurement of physical, mental, and social function domains are available from the PatientReported Outcomes Measurement Information System
(PROMIS) [9, 10]. The development of the PROMIS assessments was funded through the National Institutes of Health (NIH) Common Fund Initiative, with the purpose of providing standardized resources for use in clinical research and practice. PROMIS provides validated tools for the measurement of physical, mental, and social function domains that can be customized to capture selected domains of primary concern for specific types of patients.

Few studies have assessed these types of measures in colorectal surgery patients, and the absence of this information makes it difficult to inform patients about the near-term effects of surgery, beyond outcomes assessed by traditional clinical measures. This study was designed to provide information about the effects of colorectal surgery on physical, mental, and social well-being outcomes. We hypothesized that the PROMIS system could be utilized successfully to capture PROs in this patient population.

\section{Methods}

\section{Study population}

A 22-month (July 10, 2013 to April 30, 2015) prospective observational study was undertaken at the University of Virginia under informed consent in keeping with local Institutional Review Board standards (IRB \#16097). Adult patients (18 years of age and older) undergoing elective colorectal procedures within an enhanced recovery after surgery protocol by one of two colorectal surgeons were consented and prospectively followed by a clinical research coordinator (CRC) [11]. Patients with emergent procedures, patients less than 18 years of age, prisoners, pregnant women, and patients unable to provide consent were excluded.

\section{PRO assessment}

The PROMIS Assessment Center software was used to collect and score PROs for patients before and after surgery [12]. The assessment center software is internet-based, with the collection and secure storage of PROMIS measures and other content implemented on a study-specific website. A customized assessment instrument was developed to capture patient reported information addressing four domains: depression, pain interference, interest in sex, and ability to participate in social roles and activities. These four domains were selected for use in the study based on discussions with patients and physicians, who provided examples of psychological, symptom, and functional domains of primary concern. The assessment was limited to the collection of information for these four domains in order to minimize 
the total time required to complete the assessment during the patient's clinic visit to $10 \mathrm{~min}$ or less.

Each domain was assessed using Computer-Adaptive Testing (CAT) algorithms that present a series of health domain questions tailored to each patient, with subsequent questions selected based on a respondent's answers to prior questions [13]. The CAT-based assessments yield more precise measurement than assessments with a fixed series of questions, while requiring fewer question items than fixed length question series, and also provide improved precision at the extremes of the domain continuum [14].

The selected PROMIS domains were scored using question items with five ordinal response options (1 to 5) for each question in the domain [15]. Total scores were calculated for each domain, for each patient assessment. PROMIS domain scores are standardized to a mean of 50 and standard deviation of 10 , reflecting the distribution of scores reported for a large sample representative of the 2000 US General Census [16]. For example, a score of 40 represents a score that is one standard deviation below the reference population mean score.

Patients were identified in the outpatient clinic and approached for informed consent after the decision was made to proceed with surgery. Consenting patients accessed the assessment during clinic visits using tablet computers with wireless access to the PROMIS website [17]. Patients completed an assessment preoperatively as part of their routine preoperative workup appointment. The postoperative assessment was obtained as part of the routine postoperative follow-up appointment, which is typically scheduled for three or more weeks after discharge from the hospital following surgery.

Clinical research coordinators provided brief instruction on the use of the tablet computer to all patients and provided direct assistance with the assessment process to patients upon request. The availability of direct assistance was implemented as part of the protocol. Prior research demonstrated that while most patients would be able to complete the assessment without assistance, some patients would require direct assistance with the use of the tablet computer, with the assessment software, or with the assessment questions [17].

\section{Statistical analysis}

Longitudinal analysis of changes in PROs during the perioperative period was conducted using the multilevel random coefficient regression model (Fig. 1) [18-20]. Since measurement of the four domains is conducted both before and after surgery for each subject, the data are hierarchically structured with responses correlated among patients. Patient assessment dates also varied with regard to the lengths of time before and after surgery. The multilevel random coefficient model approach accommodates both the within subject correlation and the variation in time of assessment. Patient PRO scores are estimated as a function of time using separate intercepts and separate slopes for each patient, and the model can also include adjustments for differences in functions attributable to patient level covariates. The multilevel random coefficient model yields fixed effect estimates of the overall mean PRO score at selected time points, the difference in mean PRO score at selected time points by patient level characteristic, the change in score over time from preoperative to postoperative assessment, and the difference in the change over time by patient level characteristic.

The estimated mean scores and differences in mean scores associated with patient-level characteristics (fixed effects) were estimated with reference to the date of surgery, which is a shared longitudinal reference point for all patients. Multilevel random coefficient models were estimated to assess the overall change during the perioperative period for each of the four PRO domains. Separate multilevel random coefficient models were estimated to assess the significance of differences in these changes over time associated with patient demographics, preoperative characteristics, and postoperative outcomes. The statistical significance of the estimated fixed effects was assessed by the $F$ test statistic for type 3 tests, using the a priori selected threshold value of $p<0.05$. Figure 1 presents an example formulation of the multilevel random coefficient model.

The statistical models measure the change from pre- to post-assessment as constant over time, beginning at the time of the preoperative assessment. This aspect of the models has the potential to diminish the impact of surgery on the magnitude of the observed change in symptoms assessed at followup. We assessed the sensitivity of the model results to this assumption by recalculating results for each model, assuming that the preoperative assessment occurred on the date of surgery. The original analysis results were compared to the simulated results to determine whether any of the original model results were meaningfully different, assuming that the changes occurred between the date of surgery and the date of the postoperative assessment.

Sample size requirements for the study were estimated for a repeated measures ANOVA analysis of hypothesized levels of change in PROMIS scores during the perioperative period associated with a between-subject factor [21]. The HotellingLawley $F$ test was used to estimate sample sizes required to assess a hypothesized factor effect of a 10 unit difference in post-surgery scores from a median score of 50 prior to surgery, at alpha $=0.05$ and power $=0.80$, with correlation of residuals $=0.5$, for standard deviations of post-surgery scores of 10 or 15 units. Sample size calculations demonstrated that from 34 to 74 , patients were needed to achieve power $=0.80$ for a post-surgery score difference of 10 units associated with a given factor, at standard deviations from 10 units to 15 units, respectively. 
Fig. 1 Example level 1 and level 2 components of the combined equation represented in the multilevel random coefficient model

$$
\begin{gathered}
\text { Level } 1 \text { (Day): PRO Score }=\beta_{0 j}+\beta_{1 j}(\text { Day })+r_{i j} \\
\text { Level 2 (Patient): } \beta_{0 j}=\gamma_{00}+\gamma_{01}(\text { Sex })+u_{0 j} \\
\text { Level 2 (Patient): } \beta_{1 j}=\gamma_{10}+\gamma_{11}(\text { Sex })+u_{1 j} \\
\text { Combined: } \gamma_{00}+\gamma_{01}(\text { Sex })+\gamma_{10}(\text { Day })+\gamma_{11}(\text { Sex } * \text { Day })+\left[u_{0 j}+u_{1 j}(\text { Day })+r_{i j}\right]
\end{gathered}
$$

Fixed effect components

$\gamma_{00}$ mean PRO score at time of surgery (Day $=0$ ) for reference value of patient covariate (Sex) $\gamma_{01}$ change in PRO score (per Day) for reference value of patient covariate (Sex) $\gamma_{10}$ difference in mean PRO score (Day $=0$ ) for specified value of patient covariate (Sex) $\gamma_{11}$ difference in change in PRO score (per Day) for specified value of patient covariate (Sex)

Random effect: $\left[u_{0 j}+u_{1 j}(\right.$ Day $\left.)+r_{i j}\right]$

\section{Results}

In total, 142 patients were consented during the study period. Of these, 9 patients failed to complete the preoperative assessment, and 26 patients failed to complete the postoperative assessment. The primary reasons for failure to complete assessments were missed postoperative visits due to readmissions or other complications, cancelled surgeries, and technical difficulties with the availability of wireless access to the online assessment. There was one immediate postoperative death: An elderly patient with bilateral stomas committed suicide on postoperative day 5 within $24 \mathrm{~h}$ of discharge. Both preoperative and postoperative assessments were available for 107 patients, or $75 \%$ of the total number of colorectal surgery patients originally consented. All patients with both preoperative and postoperative assessments were included in the final study population. Figure 2 illustrates the flow of consented patients who completed or did not complete the pre-procedure and post-procedure assessments.

Differences in the distribution of demographic, preoperative, and postoperative characteristics between the subgroup of patients who did not complete the procedure assessment and the final study population were assessed for statistical significance using Fischer's exact test, with significance defined at the $p<0.05$ threshold. Patients who completed only the pre-procedure assessment were significantly different with regard to race $(p=0.0067)$, but were not significantly different with regard to the proportion male $(p=0.6662)$ or payor status $(p=0.1179)$. Surgery was cancelled following completion of the preoperative assessment for seven of the 26 patients without a post-procedure assessment.

Among the 19 patients with surgery and no post-procedure assessment, there were no statistically significant differences from the final study population in frequency of preoperative stoma, oncologic etiology, inflammatory bowel disease, diverticulitis, or receipt of neoadjuvant chemotherapy or radiation. There also were no significant differences in the frequency of surgical site infection, return to operating room, or postoperative stoma. Patients with surgery and no post-procedure assessment however had significantly lower frequencies of laparoscopic surgery (26 vs. $53 \%, p=0.0449$ ), were more likely to be readmitted following hospital discharge (38 vs. 5\%, $p<0.0001$ ), and were more likely to incur any morbidity within 30 days of surgery (37 vs. $11 \%, p=0.0096$ ). Complete results for the comparison of patients with and without post-procedure assessments are provided in a supplemental table available online.

Table 1 lists the demographic, preoperative clinical characteristics, and postoperative outcomes measured for the final study population of 107 patients with both pre- and postprocedure assessments. The majority of patients reported their race as "White" $(85 \%)$, and females accounted for slightly more than one half $(55 \%)$ of the study population. Preoperative stomas were reported in $15 \%$, inflammatory bowel disease in $19 \%$, and diverticulitis in $21 \%$. Oncologic cases represented $43 \%$, with neoadjuvant chemotherapy
Fig. 2 Illustrates the flow of preand post-procedure assessments collected for consented patients and details the number of patients who completed assessments and who did not complete assessments at each stage

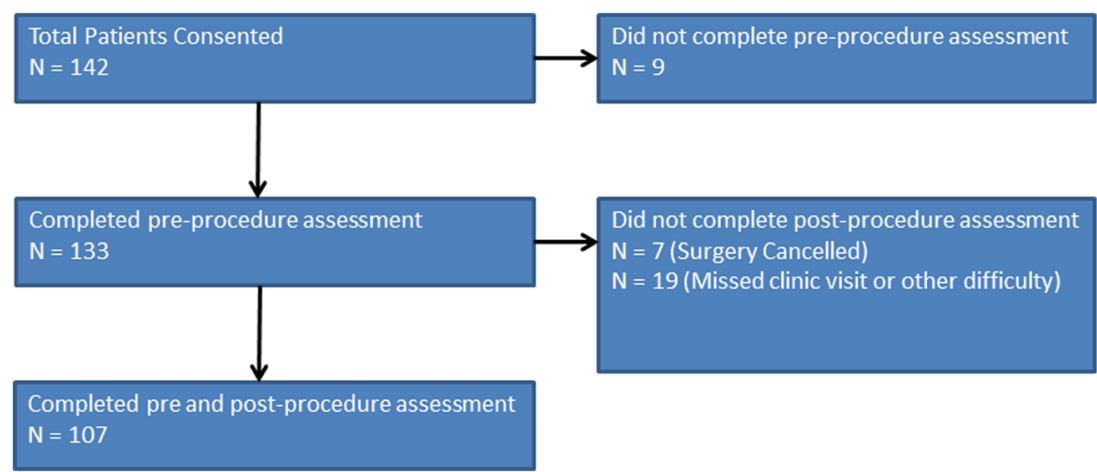


Table 1 Study population characteristics

\begin{tabular}{|c|c|c|}
\hline & Frequency & Percentage \\
\hline Total number of patients & 107 & 100.00 \\
\hline \multicolumn{3}{|l|}{ Demographics } \\
\hline Sex: male & 48 & 44.86 \\
\hline Race: Asian & 1 & 0.97 \\
\hline Race: African-American & 10 & 9.34 \\
\hline Race: White & 91 & 85.05 \\
\hline Race: not reported & 5 & 4.67 \\
\hline Insurance: Medicaid and indigent & 14 & 13.08 \\
\hline Insurance: Medicare & 40 & 37.38 \\
\hline Insurance: private & 53 & 49.53 \\
\hline \multicolumn{3}{|l|}{ Preoperative characteristics } \\
\hline Preoperative stoma: colostomy & 11 & 10.28 \\
\hline Preoperative stoma: ileostomy & 5 & 4.67 \\
\hline Preoperative stoma: none & 91 & 85.05 \\
\hline Oncologic: yes & 46 & 42.99 \\
\hline Inflammatory bowel disease: yes & 20 & 18.69 \\
\hline Diverticulitis: yes & 23 & 21.50 \\
\hline Neoadjuvant chemotherapy: yes & 27 & 25.23 \\
\hline Neoadjuvant radiation: yes & 25 & 23.36 \\
\hline \multicolumn{3}{|l|}{ Surgery } \\
\hline Laparoscopic: yes & 57 & 53.27 \\
\hline \multicolumn{3}{|l|}{ Postoperative outcomes } \\
\hline Surgical site infection: superficial & 5 & 4.67 \\
\hline Surgical site infection: deep & 1 & 0.93 \\
\hline Surgical site infection: none & 101 & 94.39 \\
\hline Returned to operating room: yes & 3 & 2.80 \\
\hline Postoperative stoma: colostomy & 11 & 10.28 \\
\hline Postoperative stoma: ileostomy & 30 & 28.04 \\
\hline Postoperative stoma: none & 66 & 61.68 \\
\hline Readmitted: yes & 5 & 4.67 \\
\hline Any morbidity with 30 days: yes & 12 & 11.21 \\
\hline
\end{tabular}

reported for $25 \%$, and neoadjuvant radiation for $23 \%$. Postoperative stomas were reported for $38 \%$. Postoperative complications included superficial or deep surgical site infections for $5 \%$, return to operating room for $3 \%$, readmission for $5 \%$, and the occurrence of any morbidity within 30 days for $11 \%$.

Table 2 lists the distributional characteristics of the PRO domain scores, the assessment completion times, the number of domain items completed, and the number of days elapsed between assessments. The mean time required to complete the assessment was $4.7 \mathrm{~min}$. Patients on average completed the preoperative assessment 1 month prior to surgery (mean 29.5 days before, $\mathrm{SD}=19.7$ ) and completed the postoperative assessment 1 month after surgery (mean 30.7 days after, $\mathrm{SD}=9.2$ ). The mean duration between the preoperative and postoperative assessment dates was 60.3 days.
Overall, the study population means for each PRO domain score were near the US general population calibrated reference means of 50, with preoperative mean scores for depression (49.9), interest in sex (47.4), pain interference (56.0), and ability to participate in social roles and activities (47.8) and with postoperative mean scores for depression (48.2), interest in sex (49.8), pain interference (56.0), and ability to participate in social roles and activities (49.4). However, the means of the preoperative pain interference scores and postoperative pain interference scores were both six points higher than the reference US general population standard.

Prior research comparing PROMIS domain scores to clinically relevant symptom severity levels demonstrates that increments of one standard deviation can be used to define reliable threshold values for grouping patients by symptom severity, such as 50-59 (mild), 60-69 (moderate), and $\geq 70$ (severe) for pain and 55-64 (mild), 65-74 (moderate), and $\geq 75$ (severe) for depression [22]. Comparison of patients grouped at these threshold values demonstrates a large improvement in the distribution of depression scores following surgery, with $12.8 \%$ of patients reporting moderate-to-severe depression at the preoperative assessment, and $4.0 \%$ reporting moderate-to-severe depression at the postoperative assessment. None of the other three domains demonstrated this large shift in score distributions. For example, the overall distribution of pain interference scores changed only slightly following surgery, with $36.5 \%$ of patients reporting moderate-tosevere pain interference at the preoperative assessment, and $33.6 \%$ reporting moderate-to-severe depression at the postoperative assessment. The proportion of patient PRO scores for each domain grouped within the ranges of 5 unit increments is provided in a supplemental table. Median, mean, and standard deviation values for each of the four PRO domains, for both the preoperative and postoperative periods, are provided for each of the demographic characteristics, preoperative clinical characteristics, and postoperative outcomes in a supplemental table available online.

The trend of change in scores during the perioperative period was assessed using the fixed effect estimates obtained by the multilevel random coefficient models. Models were estimated to assess the change in scores for each of the four PRO domains, both overall and for each demographic, preoperative clinical characteristic, and postoperative outcome. Table 3 provides a summary of the model estimated postoperative change in the mean scores, overall and for each group characteristic, along with the estimated statistical significance of the estimated change in scores. Complete results for each of the multilevel random coefficient models are provided in a supplemental table.

Overall scores for the depression domain significantly decreased (improved) over the perioperative period, with a mean estimated decline of -1.6 points per month $(p=0.03)$. Scores for the interest in sex domain increased, with a mean estimated 
Table 2 PROMIS assessment characteristics

\begin{tabular}{|c|c|c|c|}
\hline & Median & Mean & $\begin{array}{l}\text { Standard } \\
\text { deviation }\end{array}$ \\
\hline Minutes to complete total assessment & 4.00 & 4.67 & 4.11 \\
\hline Minutes to complete depression domain & 1.00 & 0.98 & 0.85 \\
\hline Minutes to complete interest in sex domain & 1.00 & 0.75 & 1.10 \\
\hline Minutes to complete pain interference domain & 1.00 & 1.53 & 1.81 \\
\hline Minutes to complete ability to participate in social roles and activities domain & 1.00 & 1.10 & 1.56 \\
\hline Depression domain items completed (range 1 to 12 ) & 4.00 & 6.59 & 3.36 \\
\hline Interest in sex domain items completed (range 1 to 4 ) & 4.00 & 3.96 & 0.34 \\
\hline Pain interference domain items completed (range 1 to 12 ) & 4.00 & 5.71 & 3.20 \\
\hline Ability to participate in social roles and activities domain completed (range 1 to 12) & 4.00 & 5.44 & 2.78 \\
\hline Days from preoperative assessment to procedure & 29.00 & 29.53 & 19.72 \\
\hline Days from procedure to postoperative assessment & 27.00 & 30.77 & 9.17 \\
\hline Days from preoperative to postoperative assessment & 56.00 & 60.29 & 22.63 \\
\hline Preoperative depression score & 49.90 & 52.20 & 11.68 \\
\hline Postoperative depression score & 48.20 & 48.27 & 10.30 \\
\hline Preoperative interest in sex score & 47.40 & 46.41 & 10.58 \\
\hline Postoperative interest in sex score & 49.80 & 48.36 & 11.59 \\
\hline Preoperative pain interference score & 56.00 & 55.41 & 10.90 \\
\hline Postoperative pain interference score & 56.00 & 54.87 & 10.55 \\
\hline Preoperative ability to participate in social roles and activities score & 47.80 & 48.44 & 10.98 \\
\hline Postoperative ability to participate in social roles and activities score & 49.40 & 49.24 & 10.73 \\
\hline
\end{tabular}

increase of 1.5 points per month $(p=0.06)$; however, the increase was not below the threshold standard for statistical significance. No statistically significant changes were demonstrated over the perioperative period in either the pain interference domain scores $(-0.18$ points, $p=0.80)$ or the ability to participate in social roles and activities domain scores $(0.44$ points, $p=0.55$ ). Figure 3 presents plots of the model estimated functions obtained for the overall change in scores during the perioperative period, for each of the four PRO domains.

Few patient characteristics were associated with statistically significant differences in the overall pattern of change in PRO domain scores following surgery. Cancer-related patient characteristics were associated with significant changes in pre-
Table 3 Estimated change in PROMIS Scores per month, overall, and by group differences

\begin{tabular}{|c|c|c|c|c|c|c|c|c|}
\hline \multirow[t]{3}{*}{ Change in score per month } & \multicolumn{8}{|c|}{ PROMIS domains } \\
\hline & \multicolumn{2}{|c|}{ Depression } & \multicolumn{2}{|c|}{ Interest in sex } & \multicolumn{2}{|c|}{$\begin{array}{l}\text { Pain } \\
\text { interference }\end{array}$} & \multicolumn{2}{|c|}{$\begin{array}{l}\text { Ability to } \\
\text { participate in social } \\
\text { roles and activities }\end{array}$} \\
\hline & Est. & $p$ value & Est. & $p$ value & Est. & $p$ value & Est. & $p$ value \\
\hline Overall & -1.62 & 0.029 & 1.55 & 0.060 & -0.18 & 0.803 & 0.44 & 0.547 \\
\hline Female vs. male & 2.08 & 0.163 & 1.43 & 0.394 & 1.43 & 0.345 & -2.06 & 0.180 \\
\hline Race all other vs. White & 2.37 & 0.212 & 1.05 & 0.586 & -1.81 & 0.349 & 1.12 & 0.578 \\
\hline Insurance other vs. private & -1.56 & 0.474 & 3.94 & 0.166 & 1.99 & 0.388 & -0.59 & 0.810 \\
\hline Medicare vs. private & 1.46 & 0.325 & 0.35 & 0.836 & -1.68 & 0.304 & 0.63 & 0.699 \\
\hline Pre-op colostomy vs. none & -3.26 & 0.129 & 0.14 & 0.960 & -2.38 & 0.278 & 2.10 & 0.349 \\
\hline Pre-op ileostomy vs. none & -2.73 & 0.364 & 3.83 & 0.208 & -2.96 & 0.360 & 2.25 & 0.476 \\
\hline Oncologic vs. other & -1.01 & 0.494 & -3.70 & 0.030 & 0.25 & 0.869 & -0.24 & 0.871 \\
\hline IBD vs. none & -3.20 & 0.100 & 1.04 & 0.662 & 0.39 & 0.850 & 1.72 & 0.406 \\
\hline Diverticulitis vs. none & 1.18 & 0.509 & 1.16 & 0.600 & -1.09 & 0.540 & 0.87 & 0.625 \\
\hline Chemo vs. none & -0.05 & 0.976 & -1.21 & 0.519 & 3.49 & 0.032 & -2.83 & 0.084 \\
\hline Radiation vs. none & -0.56 & 0.733 & -1.07 & 0.568 & 3.24 & 0.051 & -1.69 & 0.312 \\
\hline Laparoscopic vs. other & 2.21 & 0.113 & 1.78 & 0.300 & 0.24 & 0.871 & -0.86 & 0.565 \\
\hline SSI vs. none & -3.56 & 0.183 & 2.44 & 0.661 & -4.07 & 0.164 & 4.06 & 0.136 \\
\hline Operating return vs. not & -5.79 & 0.127 & -0.85 & 0.900 & -7.29 & 0.091 & 5.94 & 0.113 \\
\hline Postop colostomy vs. none & -3.41 & 0.135 & 3.28 & 0.299 & 0.30 & 0.901 & 0.77 & 0.749 \\
\hline Postop ileostomy vs. none & -3.14 & 0.055 & -3.04 & 0.113 & 1.60 & 0.346 & 1.84 & 0.294 \\
\hline Readmitted vs. not & 0.23 & 0.946 & -3.87 & 0.353 & 0.88 & 0.793 & 0.76 & 0.854 \\
\hline Any morbidity vs. none & -1.38 & 0.501 & -1.84 & 0.461 & -1.22 & 0.571 & 1.85 & 0.394 \\
\hline
\end{tabular}


Fig. 3 Panel plot depicting the linear change in patient-reported outcome measure domain scores during the perioperative period. In each plot, the solid line depicts the multilevel random coefficient model estimated linear function for the monthly change in scores, with parallel dotted lines indicating the $95 \%$ confidence interval for the linear function estimates. Hatch marks plotted along the linear function identify points in time when assessments were recorded for individual patients
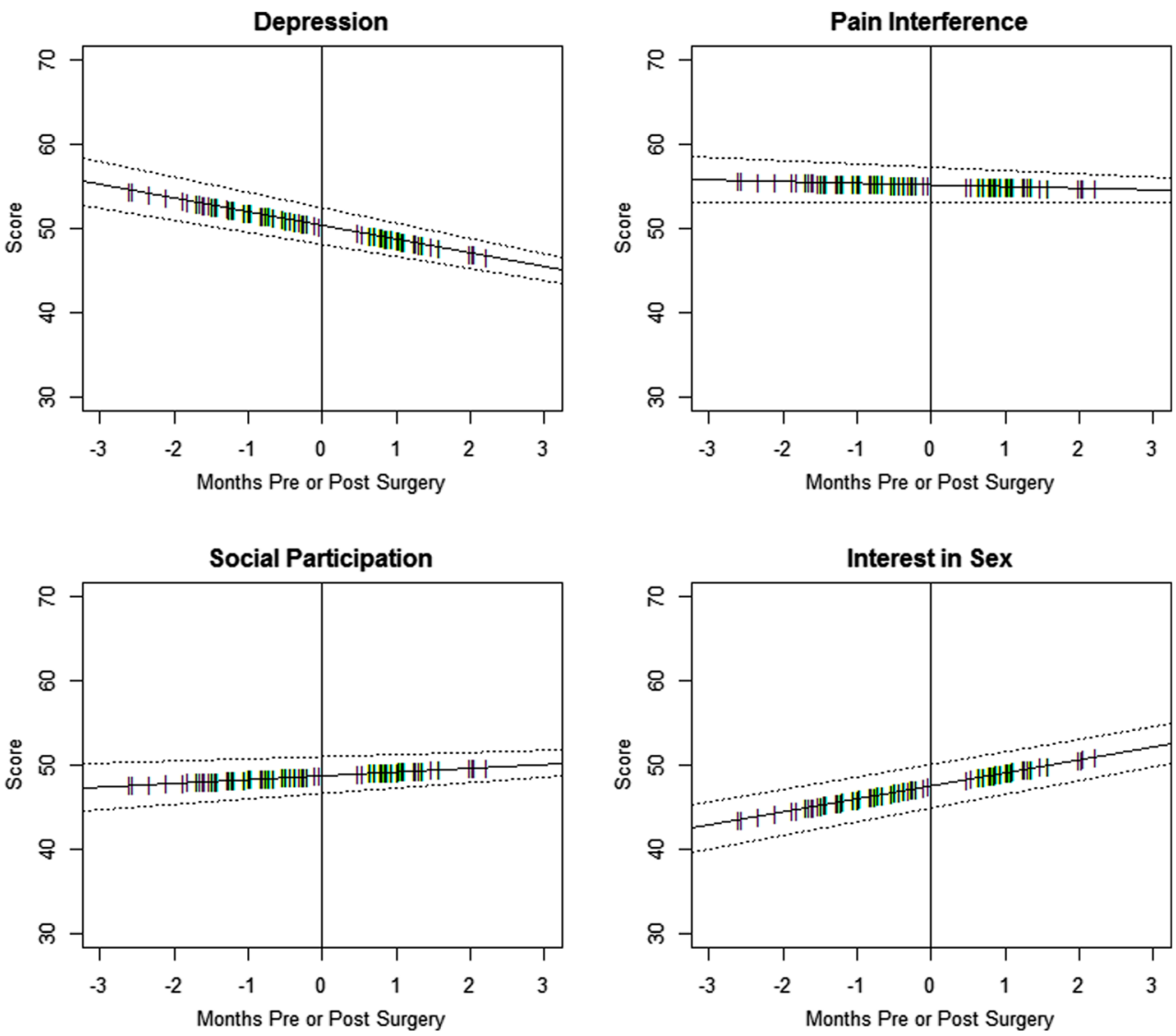

and post-procedure PRO scores. Pain interference scores for patients with neoadjuvant chemotherapy significantly increased (worsened) over the perioperative period $(+3.5$ points, $p=0.03$ ). Increased scores for pain interference reported for patients with neoadjuvant radiation were near statistical significance $(+3.2$ points, $p=0.05)$. Scores for the interest in sex domain decreased (worsened) for patients with oncologic etiology ( -3.7 points, $p=0.03$ ), compared to other patients.

Postoperative ileostomy was the only other patient characteristic associated with differences in scores large enough to be statistically significant at the $p<0.05$ threshold. Decreased depression scores were reported by patients with postoperative ileostomy, in comparison to patients without postoperative stomas, were nearly statistically significant $(-3.1$, $p=0.06$ ). Figure 4 presents plots of the estimated change in score per month during the perioperative period, for each of the four examples of group differences that were statistically significant or nearly significant at the $p<0.05$ threshold.

The observed results were not sensitive to the model assumption that change from pre- to post-assessment was constant over time. Each domain and group characteristic combination associated with a statistically significant change $(p<0.05)$ was demonstrated to also have a statistically significant change in the sensitivity analysis, where the preoperative assessment was assumed to have occurred on the day of surgery. Only one domain and group characteristic combination that was demonstrated to not be statistically significant in the original analysis (pain interference $\sim$ return to operating room, $p=0.091$ ) was statistically significant in the sensitivity analysis simulation $(p=0.048)$. Complete results for the sensitivity analysis conducted for each of the multilevel random coefficient models are provided in a supplemental table.

\section{Discussion}

This study demonstrates that PRO assessment can be conducted using the PROMIS assessment center software tools for patients undergoing major abdominal surgery in under $5 \mathrm{~min}$ during the routine clinic visit. The ready collection and availability of this information could be used to improve and inform patient care. We found that patients undergoing major colorectal surgery demonstrated no statistically significant change overall in scores for pain interference or social participation during the perioperative period, demonstrated a statistically significant decrease overall in depression scores between their pre and postoperative assessments ( -1.62 points, $p=0.03$ ), and demonstrated nearly statistically significant increases in scores for interest in sex $(+1.55$ points, $p=0.06)$.

However, cancer patients had statistically significant declines in scores for several PROs, compared to other patients. Post-procedure pain interference scores for patients with 
Fig. 4 Panel plot depicting the difference in the linear change in selected patient-reported outcome measure domain scores during the perioperative period, for four example groups. In each plot, the solid and dotted lines depict the multilevel random coefficient model estimated linear functions for the monthly change in scores, for specified groups. Hatch marks plotted along the linear function identify points in time when assessments were recorded for individual patients in each compared group
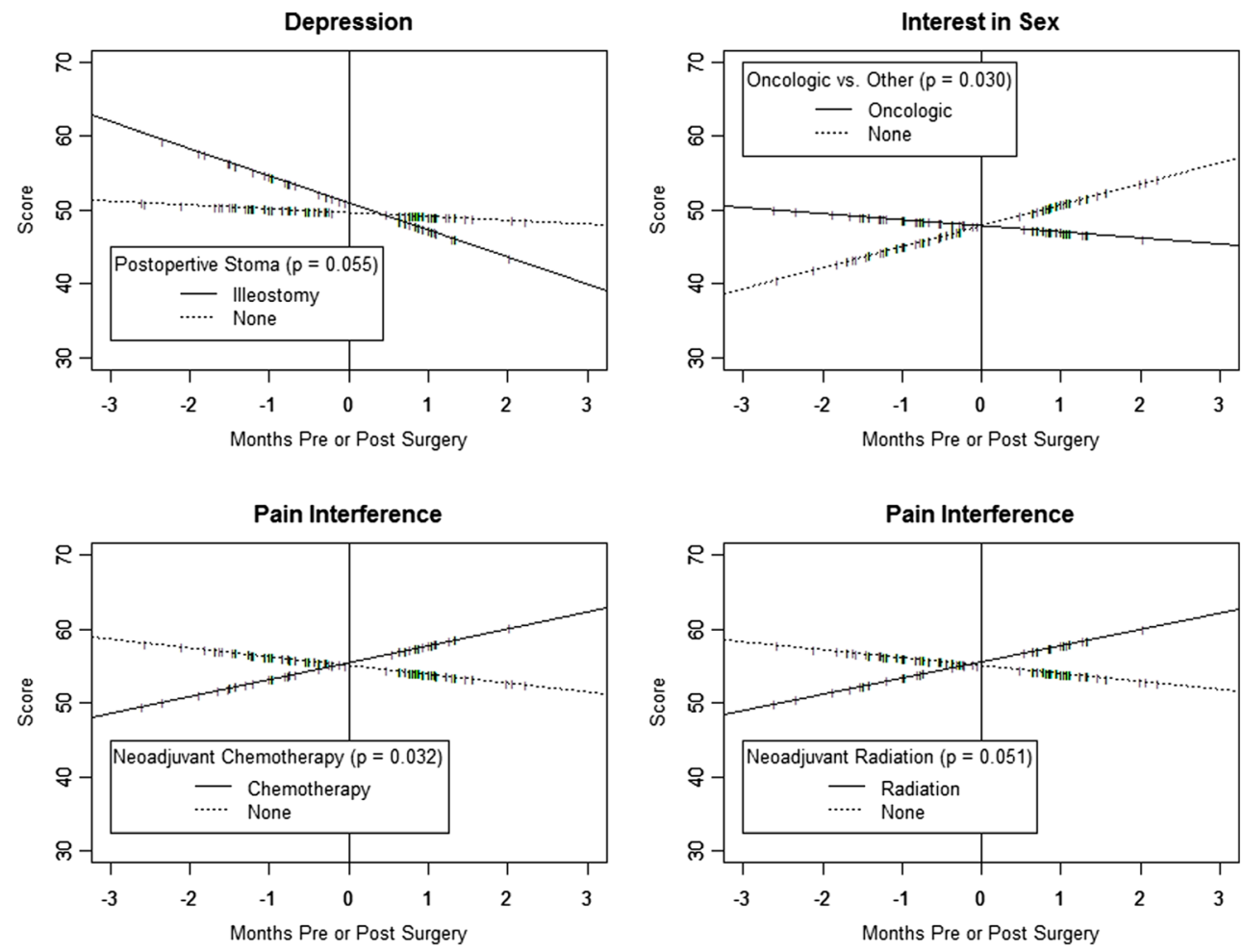

neoadjuvant chemotherapy $(+3.5$ points, $p=0.03)$ and for patients with neoadjuvant radiation $(+3.2$ points, $p=0.05$ ) were significantly increased, and scores for the interest in sex domain decreased (worsened) for patients with oncologic etiology ( -3.7 points, $p=0.03)$. These effect sizes are within the range of detectable improvements or declines in scores, and they indicate clinically significant magnitudes of change. Prior studies demonstrate that PROMIS score changes in the range from 3 to 5 points are sufficient for identifying clinically meaningful changes in pain interference and other PROMIS domains measured over time [23-25].

Other studies have assessed quality-of-life outcomes following colorectal surgery. Most recently, Brown et al., using EORTC QLQ-C30/CR38 as part of the MRC-CLASICC trial, reported that the development of complications had a negative impact on long-term quality-of-life outcomes [26]. However, to our knowledge, we are the first group to report patientreported outcomes using the PROMIS instrument in patients following major abdominal surgery. The PROMIS system is advantageous because it provides clinicians and researchers with a set of instruments that serves as the scientific foundation for patient-centered research as prioritized by the NIH. It enables researchers to customize the assessment measurements to suit the needs of the individual project while also providing a validated instrument that can be understood and shared across disciplines.

Several limitations attend this research. The study was powered to assess the significance of large differences in post-surgery scores between groups, equivalent to a difference of one standard deviation from the US general population mean. Prior research comparing PROMIS domain scores to clinically relevant symptom severity levels demonstrates that increments of one standard deviation can be used to define reliable threshold values for grouping patients by symptom severity, such as 50-59 (mild), 60-69 (moderate), and $\geq 70$ (severe) for pain and 55-64 (mild), 65-74 (moderate), and $\geq 75$ (severe) for depression [22].

While the study is adequately powered to assess the significance of large differences, assessing the significance of smaller differences associated with select patient level characteristics will require larger study populations. Given the unique nature of neoadjuvant and surgical treatment in rectal cancer patients, future studies are needed in this patient population to fully elucidate the impact of surgery on PROs. The study population included all available patients consented and receiving surgery during the 22-month study period. However, a larger study population recruited over a longer time interval would yield better estimates. The study population was recruited from a single large tertiary hospital within a standardized ERAS program, and the study population demonstrated relatively low rates of complications following surgery. The results may not be generalizable to patients receiving care in other settings or traditional care pathways. It is also important to note that all patients underwent elective surgery and were relatively healthy at baseline. These results are not generalizable to patients undergoing emergent colorectal surgery.

Post-procedure assessments were obtained for $85 \%$ of patients following surgery. However, there is a potential for a 
selection bias if patients who were struggling in the postoperative period did not complete the survey. We assessed this potential for selection bias by comparing the demographic, preoperative, and post-procedure characteristics of the subset of patients with surgery but without a post-procedure assessment to the final study population. We found that patients without post-procedure assessments were more likely to have reported any morbidity 30 days following surgery and were more likely to have been readmitted following discharge. Patients without post-procedure assessments also had significantly lower frequencies of laparoscopic surgery, which is associated with fewer complications and quicker recovery than open surgical procedures [27]. These comparisons suggest that post-procedure assessments were not obtained for some patients because of follow-up schedule changes and other exigencies related to complications and readmissions following surgery.

Another limitation of the study is that assessment results were obtained only for the immediate perioperative period. Longer term follow-up of patients, while requiring additional resources and different protocols for scheduling assessment data collection, would provide important information about patient outcomes. Examining patient outcomes over longer periods of time following surgery may also produce different results regarding the relationships between demographic, preprocedure, and post-procedure events. Extending the followup period to include additional longitudinal assessment points could also reduce the number patients with missing postprocedure assessments, by providing additional opportunities to collect PROs at later follow-up appointments.

Finally, we focused the pilot study on four domains (depression, pain interference, interest in sex, and ability to function socially) that were selected following discussions with stakeholders. We selected only four domains in order to minimize the time required to complete the assessment. At the conclusion of the study, we learned that most patients were able to complete the assessment in less than $5 \mathrm{~min}$. Several other domains are of great importance, including information about gastrointestinal symptoms, fatigue, pain intensity, and anxiety. Our results suggest that the number of domains included in the assessment protocol could be expanded to include several additional domains of interest, without increasing the duration of the assessment beyond $10 \mathrm{~min}$ for most patients.

\section{Conclusions}

Recovery from surgery is a complicated process that has effects on the physical, emotional, and social domains of health. Patients may experience a variety of emotions, ranging from depression, due to the loss of autonomy, to anxiety at not knowing what will happen next. There are also social challenges that result from the loss of independence following major surgery. These data suggest that the majority of patients quickly return to baseline physical, mental, and social function following colorectal surgery. This information is essential for preoperative patient counseling about the typical impact of colorectal surgery on quality of life. Further studies are needed to validate our results in a broader population, to provide information about other domains of key interest, and to more closely examine the effect of individual characteristics (such as diagnosis and surgical type) and the development of complications on postoperative changes in PROs among colorectal surgery patients.

Acknowledgements PROMIS $®$ was funded with cooperative agreements from the National Institutes of Health (NIH) Common Fund Initiative (U54AR057951, U01AR052177, U54AR057943, U54AR057926, U01AR057948, U01AR052170, U01AR057954, U01AR052171, U01AR052181, U01AR057956, U01AR052158, U01AR057929, U01AR057936, U01AR052155, U01AR057971, U01AR057940, U01AR057967, U01AR052186). The contents of this article uses data developed under PROMIS. These contents do not necessarily represent an endorsement by the US Federal Government or PROMIS. See www.nihpromis.org for additional information on the PROMIS initiative.

Contributions Each author has made substantial contributions to conception and design, or acquisition of data, or analysis and interpretation of data.

\section{Compliance with ethical standards}

Conflict of interest The authors declare that there are no conflicts of interest to report.

Dr. Stukenborg retains full control of all primary data used in this analysis and the authors agree to allow the journal to review the data if requested. The authors have no possible conflicts of interest in the manuscript, including no financial, consultant, institutional, and other relationships that might lead to bias.

Open Access This article is distributed under the terms of the Creative Commons Attribution-NonCommercial 4.0 International License (http:// creativecommons.org/licenses/by-nc/4.0/), which permits any noncommercial use, distribution, and reproduction in any medium, provided you give appropriate credit to the original author(s) and the source, provide a link to the Creative Commons license, and indicate if changes were made.

\section{References}

1. Temple L, Fuzesi S, Patil S (2009) The importance of determining quality of life in clinical trials. Surgery 145:622-626

2. Basch E, Torda P, Adams K (2013) Standards for patient-reported outcome-based performance measures. JAMA 310:139-140

3. Basch E, Abernethy AP, Mullins CD, Reeve BB, Smith ML, Coons SJ, Sloan J, Wenzel K, Chauhan C, Eppard W, Frank ES, Lipscomb J, Raymond SA, Spencer M, Tunis S (2012) Recommendations for incorporating patient-reported outcomes into clinical comparative effectiveness research in adult oncology. J Clin Oncol 30:42494255

4. Franklin PD, Lewallen D, Bozic K, Hallstrom B, Jiranek W, Ayers DC (2014) Implementation of patient-reported outcome measures 
in U.S. Total joint replacement registries: rationale, status, and plans. J Bone Joint Surg Am 96(Suppl 1):104-109

5. Berliner JL, Brodke DJ, Chan V, SooHoo NF, Bozic KJ (2016) John Charnley award: preoperative patient-reported outcome measures predict clinically meaningful improvement in function after THA. Clin Orthop Relat Res 474:321-329

6. Federal Register. Vol. 80, No. 134.Tuesday, July 14, 2015. Proposed rules. Medicare program; Comprehensive care for joint replacement payment model for Acute Care Hospitals Furnishing Lower Extremity Joint Replacement Services. Section III.C.5.b.(2)

7. Bilimoria KY, Cella D, Butt Z (2014) Current challenges in using patient-reported outcomes for surgical care and performance measurement: everybody wants to hear from the patient, but are we ready to listen? JAMA Surgery 149:505-506

8. McNair A, Whistance RN, Forsythe RO, Rees J, Jones JE, Pullyblank AM, Avery K, Brookes ST, Thomas MG, Sylvester PA, Russell A, Oliver A, Morton D, Kennedy R, Jayne DG, Huxtable R, Hackett R, Dutton SJ, Coleman MG, Card M, Brown J, Blazeby JM (2015) Synthesis and summary of patient-reported outcome measures (PROMs) to inform the development of a core outcome set in colorectal cancer surgery. Color Dis 17:217-229

9. Garcia SF, Cella D, Clauser SB, Flynn KE, Lad T, Lai JS, Reeve BB, Smith AW, Stone AA, Weinfurt K (2007) Standardizing patient-reported outcomes assessment in cancer clinical trials: a patient-reported outcomes measurement information system initiative. J Clin Oncol 25:5106-5112

10. Cella D, Riley W, Stone A, Rothrock N, Reeve B, Yount S, Amtmann D, Bode R, Buysse D, Choi S, Cook K, Devellis R, DeWalt D, Fries JF, Gershon R, Hahn EA, Lai JS, Pilkonis P, Revicki D, Rose M, Weinfurt K, Hays R (2010) The patientreported outcomes measurement information system (PROMIS) developed and tested its first wave of adult self-reported health outcome item banks: 2005-2008. J Clin Epidemiol 63:1179-1194

11. Thiele RH, Rea KM, Turrentine FE, Friel CM, Hassinger TE, McMurry TL, Goudreau BJ, Umapathi BA, Kron IL, Sawyer RG, Hedrick TL (2015) Standardization of care: impact of an enhanced recovery protocol on length of stay, complications, and direct costs after colorectal surgery. J Am Coll Surg 220:430-443

12. Gershon RC, Rothrock N, Hanrahan R, Bass M, Cella D (2010) The use of PROMIS and assessment center to deliver patientreported outcome measures in clinical research. Journal of applied measurement 11:304-314

13. Revicki DA, Cella DF (1997) Health status assessment for the twenty-first century: item response theory, item banking and computer adaptive testing. Qual Life Res 6:595-600

14. Hung M, Stuart AR, Higgins TF, Saltzman CL, Kubiak EN (2014) Computerized adaptive testing using the PROMIS physical function item bank reduces test burden with less ceiling effects compared with the short musculoskeletal function assessment in orthopaedic trauma patients. J Orthop Trauma 28:439-443

15. PROMIS Scoring Manuals. Patient reported outcomes measurement information system. Assessment Center. Accessed on 19 Jan 2016

16. Liu H, Cella D, Gershon R, Shen J, Morales LS, Riley W, Hays RD (2010) Representativeness of the patient-reported outcomes measurement information system internet panel. J Clin Epidemiol 63: $1169-1178$

17. Stukenborg GJ, Blackhall L, Harrison J, Barclay JS, Dillon P, Davis MA, Mallory M, Read P (2014) Cancer patient-reported outcomes assessment using wireless touch screen tablet computers. Qual Life Res 23:1603-1607

18. Laird NM, Ware JH (1982) Random-effects models for longitudinal data. Biometrics 38:963-974

19. Rajeswaran J, Blackstone EH (2015) Patient-reported outcomes and importance of their appropriate statistical analyses. $\mathrm{J}$ Thorac Cardiovasc Surg 150:461-462

20. Detry MA, Ma Y (2016) Analyzing repeated measurements using mixed models. JAMA 315:407-408

21. Guo Y, Logan HL, Glueck DH, Muller KE (2013) Selecting a sample size for studies with repeated measures. BMC Med Res Methodol 13:100

22. Cella D, Choi S, Garcia S, Cook KF, Rosenbloom S, Lai J-S, Tatum DS, Gershon R (2014) Setting standards for severity of common symptoms in oncology using the PROMIS item banks and expert judgment. Qual Life Res 23:2651-2661

23. Jensen RE, Moinpour CM, Potosky AL, Lobo T, Hahn EA, Hays RD, Cella D, Smith AW, Wu XC, Keegan TH, Paddock LE, Stroup AM, Eton DT (2016) Responsiveness of 8 patient-reported outcomes measurement information system (PROMIS) measures in a large, community-based cancer study cohort. Cancer 123:327-335

24. Yost KJ, Eton DT, Garcia SF, Cella D (2011) Minimally important differences were estimated for six patient-reported outcomes measurement information system - cancer scales in advanced-stage cancer patients. J Clin Epidemiol 64:507-516

25. Hays RD, Spritzer KL, Fries JF, Krishnan E (2015) Responsiveness and minimally important difference for the patient-reported outcomes measurement information system (PROMIS) 20-item physical functioning short form in a prospective observational study of rheumatoid arthritis. Ann Rheum Dis 74:104-107

26. Brown SR, Mathew R, Keding A, Marshall HC, Brown JM, Jayne DG (2014) The impact of postoperative complications on long-term quality of life after curative colorectal cancer surgery. Ann Surg 259:916-923

27. King PM, Blazeby JM, Ewings P, Longman RJ, Kipling RM, Franks PJ, Sheffield JP, Evans LB, Soulsby M, Bulley SH, Kennedy RH (2006) The influence of an enhanced recovery programme on clinical outcomes, costs and quality of life after surgery for colorectal cancer. Color Dis 8:506-513 